\title{
Contact frequency determines outcome of basal insulin initiation trials in type 2 diabetes
}

\author{
S. G. H. A. Swinnen • J. H. DeVries
}

Received: 24 April 2009 / Accepted: 10 August 2009 /Published online: 8 September 2009

(C) The Author(s) 2009. This article is published with open access at Springerlink.com

\begin{abstract}
Aims/hypothesis The aim of the present study was to investigate whether predetermined contact frequency with the study team and endpoint insulin dose are associated with study outcomes in basal insulin initiation trials in type 2 diabetes.

Methods A systematic Medline search was performed. Using data from the selected studies, contact frequency was plotted against $\mathrm{HbA}_{1 \mathrm{c}}$ reduction and endpoint insulin dose. The importance of face-to-face vs telephone contact was also analysed. Insulin dose was plotted against $\mathrm{HbA}_{1 \mathrm{c}}$ reduction, hypoglycaemia rate and weight gain. To investigate non-specific study effects, the relationship between contact frequency and $\mathrm{HbA}_{1 \mathrm{c}}$ was also assessed in dipeptidyl peptidase-4 (DPP-4) inhibitor trials.

Results The reduction in $\mathrm{HbA}_{1 \mathrm{c}}$ was highly correlated with contact frequency and endpoint insulin dose $\left(r^{2}=0.751\right.$, $p<0.001$ and $r^{2}=0.433, p=0.008$, respectively). However, after adjusting for contact frequency, the relationship between insulin dose and $\mathrm{HbA}_{1 \mathrm{c}}$ reduction was no longer significant $(p=0.270)$. The frequency of both clinical and telephone contacts were independent predictors of $\mathrm{HbA}_{1 \mathrm{c}}$ improvement ( $p=0.010$ and $p<0.001$, respectively). We found no doseresponse relationship between end-of-study insulin dose and hypoglycaemia or weight gain. In DPP-4 inhibitor studies, contact frequency was not positively associated with $\mathrm{HbA}_{1 \mathrm{c}}$. Conclusions/interpretation The frequency of contact with the study team is highly correlated with the improvement in $\mathrm{HbA}_{1 \mathrm{c}}$ achieved in basal insulin initiation trials in type 2 diabetic patients. This has important implications for trial design and interpretation, as well as for clinical care.
\end{abstract}

S. G. H. A. Swinnen $(\bowtie) \cdot$ J. H. DeVries

Department of Internal Medicine, Academic Medical Centre,

Meibergdreef 9,

1105 AZ Amsterdam, the Netherlands

e-mail: s.g.swinnen@amc.uva.nl
Keywords Basal insulin preparations · Glycaemic control . Insulin initiation · Insulin therapy · Type 2 diabetes
Abbreviations
DPP-4 Dipeptidyl peptidase-4
RCT Randomised controlled trial
SMBG Self-monitoring of blood glucose

\section{Introduction}

In type 2 diabetes basal insulin preparations are advocated for the initiation of insulin therapy [1]. Insulin initiation using insulin detemir (NN304) [B29Lys(E-tetradecanoyl),desB30 human insulin] or insulin glargine (A21Gly,B31Arg,B32Arg human insulin) has been examined in a number of Phase 3 and 4 clinical studies [2-8], comparing either analogue with $\mathrm{NPH}$ insulin or with each other [6] for their ability to decrease $\mathrm{HbA}_{1 \mathrm{c}}$ levels. However, in addition to the merits of the insulin preparation under investigation, factors related to the design of the trials may affect this and other study endpoints. Therefore, we explored whether frequency of contact with the study team as per study protocol and endpoint insulin dose are associated with study outcomes in randomised controlled trials (RCTs) comparing insulin initiation with one basal insulin vs another in insulin-naive participants with type 2 diabetes.

\section{Methods}

Medline was searched using the terms 'detemir', 'glargine', 'neutral protamine hagedorn', 'NPH', 'neutral protamine lispro', 'NPL', 'type 2 diabetes', 'non-insulin dependent diabetes mellitus' and 'NIDDM'. In order to take the current more stringent glycaemic goals and the relatively 
recent use of insulin titration algorithms into account, the search was limited to studies published between April 1999 and April 2009. All English-language RCTs with a duration $\geq 24$ weeks comparing insulin initiation with a basal insulin vs another basal insulin in insulin-naive type 2 diabetic participants were included. Studies investigating combination therapy with rapid-acting insulin and those that had differences across treatment arms in additional glucose-lowering interventions (e.g. oral agents), titration algorithms and/or contact frequency were excluded.

The search yielded 417 papers, including 112 RCTs, of which ten met our criteria [2-11]. Using these, associations between the frequency of contact as per protocol and the study outcomes reduction in $\mathrm{HbA}_{1 \mathrm{c}}$ and endpoint daily insulin dose were explored by plotting the contact frequency (clinical and telephone contacts combined and standardised to the number of contacts per year) against the two outcome measures. To examine the relative importance of clinical vs telephone contact, we standardised both to the number of contacts per year and performed a multivariable linear regression analysis. Similarly, endpoint insulin dose, related to the study design factors titration frequency and titration target, was plotted against the study outcomes $\mathrm{HbA}_{1 \mathrm{c}}$ reduction, hypoglycaemia rate and weight gain. For all associations, we performed weighted least squares regression using treatment group size as the weight variable.

Of the ten included studies, two were excluded from all analyses as they did not report contact frequency, endpoint insulin dose as $\mathrm{U} \mathrm{kg}^{-1} \mathrm{day}^{-1}$ or endpoint weight $[2,11]$. One more study did not mention the frequency of contact [9]. Three more studies did not report insulin dose as $\mathrm{U} \mathrm{kg}^{-1}$ day $^{-1}$ but for two this could be calculated [3, 4]. For another three studies the change in $\mathrm{HbA}_{1 \mathrm{c}}$ was calculated $[5,7,8]$. Concerning hypoglycaemia, the studies used variable definitions. We used event rates rather than numbers of participants affected to account for the effect of study duration on hypoglycaemia frequency. We defined hypoglycaemia as an event confirmed by a low glucose measurement, as this definition maximised the number of studies that could be included in the analysis $[4-7,9]$. All in all, 15 treatment groups were analysed for the associations between contact frequency and $\mathrm{HbA}_{1 \mathrm{c}}$, dose and $\mathrm{HbA}_{1 \mathrm{c}}$, and dose and weight gain; 13 were analysed for the association between contact frequency and dose, and ten for the association between dose and hypoglycaemia.

In addition to the efficacy of the study insulin and certain features of the study design, improvements in $\mathrm{HbA}_{1 \mathrm{c}}$ during trial participation may result from non-specific study effects $[12,13]$. To investigate these non-specific study benefits, we also determined the relationship between contact frequency and $\mathrm{HbA}_{1 \mathrm{c}}$ reduction in studies examining the initiation of dipeptidyl peptidase-4 (DPP-4) inhibitors. Both study populations chose to start a new intervention in the setting of a clinical trial, so presumably all were equally motivated to improve their diabetes management. However, in contrast with the continuous dose titration during the insulin trials, fixed dosages were used throughout the DPP-4 studies. We included the trials that were recently meta-analysed in a Cochrane systematic review and that compared DPP-4 inhibitor monotherapy with placebo or a single oral glucose-lowering agent [14]. After exclusion of one study because of lack of contact frequency data, we analysed 14 studies comprising 29 DPP-4 inhibitor treatment groups.

\section{Results}

Figure 1a shows that the improvement in $\mathrm{HbA}_{1 \mathrm{c}}$ achieved in studies of basal insulin initiation in patients with type 2 diabetes was highly correlated with the predetermined frequency of contact with the study team $\left(r^{2}=0.751\right.$, $p<0.001)$. Multivariable analysis of the standardised numbers of clinical visits and telephone contacts showed a strong association with $\mathrm{HbA}_{1 \mathrm{c}}$ reduction (adjusted $r^{2}$ value 0.754). Both frequencies were independent predictors of $\mathrm{HbA}_{1 \mathrm{c}}$ improvement $(p=0.010$ and $p<0.001$ for clinical and telephone contacts, respectively). The regression equation was: $\mathrm{HbA}_{1 \mathrm{c}}$ reduction $=0.282+(0.033 \times$ number of clinical contacts per year $)+(0.055 \times$ number of telephone contacts per year). In the DPP-4 studies, however, higher contact frequency was not, and, if anything, negatively associated with greater improvement in glycaemic control $\left(r^{2}=0.233\right.$, standardised $\beta=-0.483, p=0.008$ ) (Fig. 1f).

In the insulin trials, we also found significant relationships between contact frequency and insulin dose at study endpoint $\left(r^{2}=0.366, p=0.028\right)$ (Fig. 1b), and between endpoint insulin dose and $\mathrm{HbA}_{1 \mathrm{c}}$ reduction $\left(r^{2}=0.433, p=0.008\right)$ (Fig. 1c). The effect of increasing insulin doses on the occurrence of hypoglycaemia was not apparent in insulin-naive patients $\left(r^{2}=0.011, p=0.774\right)$, and we found no evidence for a doseresponse relationship between insulin dose and weight gain $\left(r^{2}=0.076, p=0.320\right)$ (Fig. 1d, e). Finally, to determine whether contact frequency and insulin dose were also independently associated with improvement in glycaemic control, we performed a multivariable regression analysis. The adjusted $r^{2}$ value of this model was 0.718 , and while contact frequency remained an independent predictor of $\mathrm{HbA}_{1 \mathrm{c}}$ reduction $(p=0.003)$, insulin dose did not $(p=0.270)$.

\section{Discussion}

Our main finding was that, while there were significant dose-response relationships between the predetermined frequency of contact and endpoint insulin dose and the 

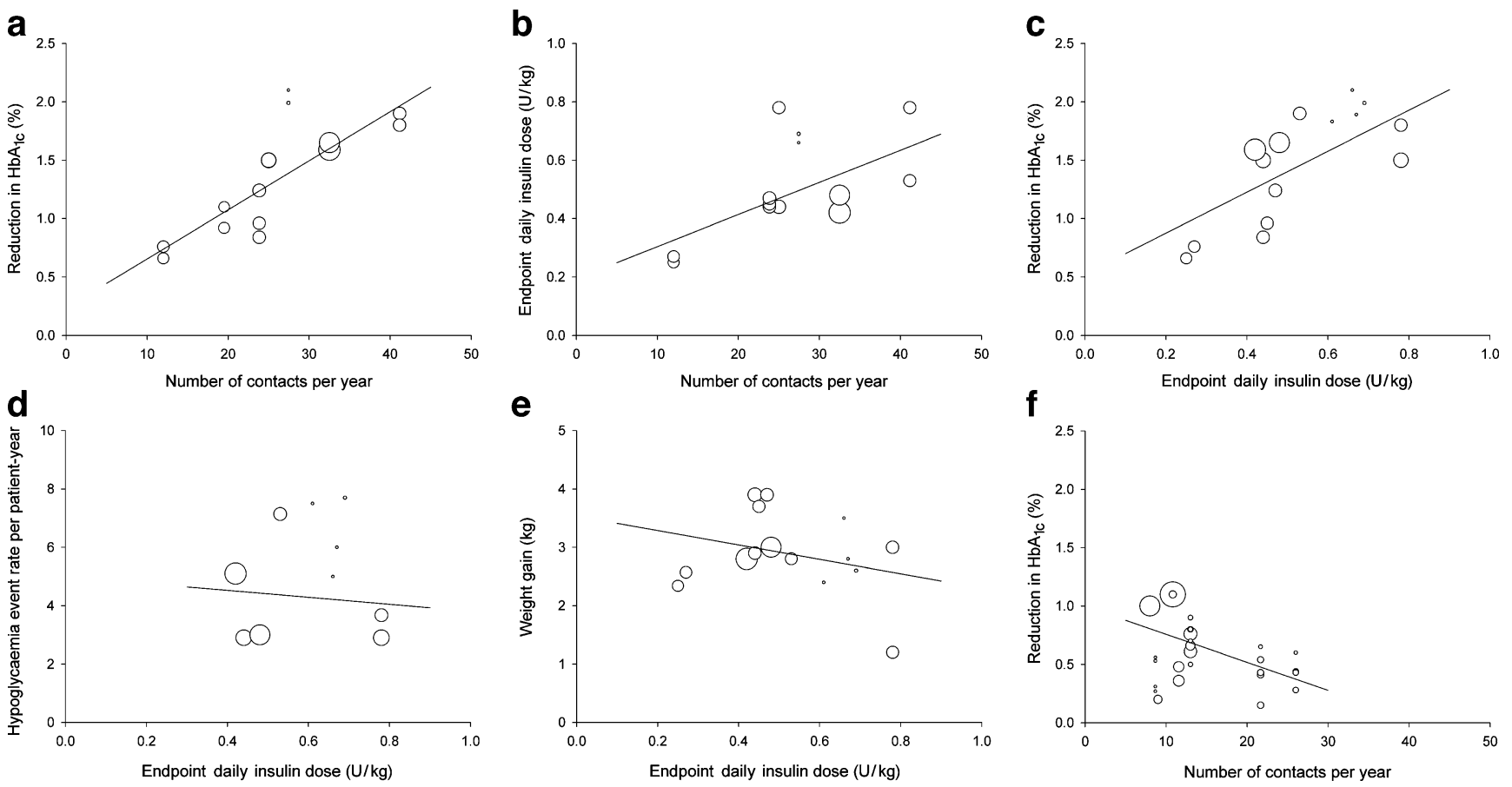

Fig. 1 Relationships between (a) the frequency of contact with the study team as per study protocol and mean reduction in $\mathrm{HbA}_{1 \mathrm{c}}$ level (15 treatment groups, $r^{2}=0.751$, standardised $\beta=0.866, p<0.001$ ), and (b) the frequency of contact and mean end-of-study daily insulin dose (13 treatment groups, $r^{2}=0.366$, standardised $\beta=0.605, p=0.028$ ). Relationships between mean endpoint daily insulin dose and (c) mean reduction in $\mathrm{HbA}_{1 \mathrm{c}}$ level ( 15 treatment groups, $r^{2}=0.433$, standardised $\beta=0.658, p=0.008$ ), (d) rate of hypoglycaemia confirmed by a low glucose measurement per patient-year (ten treatment groups,

improvement in glycaemic control achieved during RCTs investigating basal insulin initiation in type 2 diabetic patients, only contact frequency was an independent predictor of $\mathrm{HbA}_{1 \mathrm{c}}$ reduction. The frequency of both clinical visits and telephone contacts showed a significant relationship with $\mathrm{HbA}_{1 \mathrm{c}}$ improvement, but a higher frequency of telephone contact may be particularly beneficial. In studies examining the start of DPP-4 inhibitors we did not find a positive association between contact frequency and $\mathrm{HbA}_{1 \mathrm{c}}$ improvement.

Assuming that the non-specific study effects of the insulin and DPP-4 trials were comparable, the lack of a logical association between contact frequency and $\mathrm{HbA}_{1 \mathrm{c}}$ improvement in the latter studies suggests that the benefit of frequent patient contact found in the insulin trials is primarily related to the frequency of insulin dose titration. This is supported not only by Fig. 1b, which shows a significant relationship between contact frequency and insulin doses used at study endpoint, but also by the multivariable analysis of the frequencies of clinical and telephone contacts. The higher $\beta$ coefficient for telephone contacts suggests that these may be more beneficial than clinical visits, presumably due to their focus on dose $r^{2}=0.011$, standardised $\left.\beta=-0.104, p=0.774\right)$ and (e) mean weight gain (15 treatment groups, $r^{2}=0.076$, standardised $\beta=-0.276$, $p=0.320$ ). RCTs comparing insulin initiation with a basal insulin vs another basal insulin in insulin-naive participants with type 2 diabetes were analysed. $\mathbf{f}$ Relationship between the predetermined frequency of contact and mean reduction in $\mathrm{HbA}_{1 \mathrm{c}}$ level in clinical studies investigating initiation of DPP-4 inhibitor treatment (29 treatment groups, $r^{2}=0.233$, standardised $\beta=-0.483, p=0.008$ ). The size of the symbols reflects treatment group size

titration (compared with the performance of many studyrelated procedures during clinical visits).

However, it cannot be ruled out that the non-specific study benefits did indeed differ between the two types of trials. In type 1 diabetes, the improvement in glycaemic control after trial participation itself was found to be mediated by increased self-monitoring of blood glucose (SMBG) and a more active coping style [13]. In contrast with the insulin initiation trials, SMBG was not performed in the DPP-4 studies. Additionally, it is possible that patients failing on oral therapy and requiring insulin are more 'ready for change' and more motivated to do well than those (merely) starting on another tablet. However, regardless of whether the benefit of regular patient contact is primarily related to titration frequency or to non-specific study effects, our analyses demonstrate that predetermined contact frequency is a major determinant of the $\mathrm{HbA}_{1 \mathrm{c}}$ improvement achieved in insulin initiation trials. In addition to the obvious consequences for the design and interpretation of clinical trials comparing different insulin preparations, our findings have important implications for patient care, in as far as providing frequent contact after starting insulin therapy may be very effective in improving glycaemic control. 
We found no relationship between daily insulin dose and hypoglycaemia event rate, but this may reflect the low a priori risk of hypoglycaemia of the study populations. These type 2 diabetic participants had just started insulin treatment, so were presumably protected against hypoglycaemia by residual endogenous insulin secretion. An alternative possible explanation for the observed lack of a relationship is that participants on higher daily doses after titration have similar rates of hypoglycaemia to those in whom titration is stopped at lower insulin doses. In this case, the graph could suggest that perceived risk of hypoglycaemia is the signal to stop insulin dose titration. The lack of association between insulin dose and weight gain suggests that the weight increase commonly seen after initiation of insulin therapy is related to reductions in glucosuria and/or increased energy intake, rather than to the number of insulin injections or the daily insulin dose [15].

In conclusion, the frequency of contact with the study team is highly correlated with the improvement in glycaemic control achieved after basal insulin initiation in type 2 diabetes. Our analyses indicate that when comparing the outcomes of different clinical trials, their design should also be considered. Our findings also have implications for trial design and patient care. Frequent contact and dose titration may facilitate successful insulin initiation.

Duality of interest S. G. H. A. Swinnen is employed by the Department of Internal Medicine of the Academic Medical Centre, partly through funding from Novo Nordisk and sanofi-aventis for the conduct of clinical trials. J. H. DeVries has received honoraria for consultancy work as well as research funding from Novo Nordisk and sanofi-aventis.

Open Access This article is distributed under the terms of the Creative Commons Attribution Noncommercial License which permits any noncommercial use, distribution, and reproduction in any medium, provided the original author(s) and source are credited.

\section{References}

1. Nathan DM, Buse JB, Davidson MB et al (2009) Medical management of hyperglycemia in type 2 diabetes: a consensus algorithm for the initiation and adjustment of therapy: a consensus statement of the American Diabetes Association and the European Association for the Study of Diabetes. Diabetes Care 32:193-203
2. Eliaschewitz FG, Calvo C, Valbuena H et al (2006) Therapy in type 2 diabetes: insulin glargine vs NPH insulin both in combination with glimepiride. Arch Med Res 37:495-501

3. Fritsche A, Schweitzer MA, Häring HU (2003) Glimepiride combined with morning insulin glargine, bedtime neutral protamine hagedorn insulin, or bedtime insulin glargine in patients with type 2 diabetes. Ann Intern Med 138:952-959

4. Hermansen K, Davies M, Derezinski T et al (2006) A 26-week, randomized, parallel, treat-to-target trial comparing insulin detemir with NPH insulin as add-on therapy to oral glucose-lowering drugs in insulin-naive people with type 2 diabetes. Diabetes Care 29:1269-1274

5. Riddle MC, Rosenstock J, Gerich J (2003) The treat-to-target trial: randomized addition of glargine or human NPH insulin to oral therapy of type 2 diabetic patients. Diabetes Care 26:3080-3086

6. Rosenstock J, Davies M, Home P, Larsen J, Koenen C, Schernthaner G (2008) A randomised, 52-week, treat-to-target trial comparing insulin detemir with insulin glargine when administered as add-on to glucose-lowering drugs in insulinnaïve people with type 2 diabetes. Diabetologia 51:408-416

7. Yki-Järvinen H, Kauppinen-Mäkelin R, Tiikkainen M et al (2006) Insulin glargine or NPH combined with metformin in type 2 diabetes: the LANMET study. Diabetologia 49:442-451

8. Yki-Järvinen H, Dressler A, Ziemen M, HOE 901/3002 study group (2000) Less nocturnal hypoglycemia and better post-dinner glucose control with bedtime insulin glargine compared with bedtime NPH insulin during insulin combination therapy in type 2 diabetes. Diabetes Care 23:1130-1136

9. Esposito K, Ciotola M, Maiorino MI et al (2008) Addition of neutral protamine lispro insulin or insulin glargine to oral type 2 diabetes regimens for patients with suboptimal glycemic control: a randomized trial. Ann Intern Med 149:531-539

10. Pan CY, Sinnassamy P, Chung KD, Kim KW (2007) Insulin glargine vs NPH insulin therapy in Asian type 2 diabetes patients. Diabetes Res Clin Pract 76:111-118

11. Standl E, Maxeiner S, Raptis S, HOE 901/4009 study group (2006) Once-daily insulin glargine administration in the morning compared to bedtime in combination with morning glimepiride in patients with type 2 diabetes: an assessment of treatment flexibility. Horm Metab Res 38:172-177

12. Gale EAM, Beattie SD, Hu J, Koivisto V, Tan MH (2007) Recruitment to a clinical trial improves glycemic control in patients with diabetes. Diabetes Care 30:2989-2992

13. DeVries JH, Snoek FJ, Kostense PJ, Heine RJ (2003) Improved glycaemic control in type 1 diabetes patients following participation per se in a clinical trial-mechanisms and implications. Diabetes Metab Res Rev 19:357-362

14. Richter B, Bandeira-Echtler E, Bergerhoff K, Lerch CL (2008) Dipeptidyl peptidase-4 (DPP-4) inhibitors for type 2 diabetes mellitus. Cochrane Database Syst Rev, Issue 2, Art. no. CD006739. doi:10.1002/14651858.CD006739.pub2

15. Yki-Järvinen $H$ (2001) Combination therapies with insulin in type 2 diabetes. Diabetes Care 24:758-767 